\title{
Weighted Multilinear Hardy Operators on Herz Type Spaces
}

\author{
Shuli Gong, ${ }^{1}$ Zunwei Fu, ${ }^{2}$ and Bolin $\mathrm{Ma}^{3}$ \\ ${ }^{1}$ College of Mathematics and Econometrics, Hunan University, Changsha, Hunan 410082, China \\ ${ }^{2}$ Department of Mathematics, Linyi University, Linyi, Shandong 276005, China \\ ${ }^{3}$ College of Mathematics Physics and Information Engineering, Jiaxing University, Jiaxing, Zhejiang 314001, China
}

Correspondence should be addressed to Zunwei Fu; zwfu@mail.bnu.edu.cn

Received 23 November 2013; Accepted 17 December 2013; Published 5 January 2014

Academic Editors: M. M. Cavalcanti and S. Mohiuddine

Copyright (C) 2014 Shuli Gong et al. This is an open access article distributed under the Creative Commons Attribution License, which permits unrestricted use, distribution, and reproduction in any medium, provided the original work is properly cited.

\begin{abstract}
This paper focuses on the bounds of weighted multilinear Hardy operators on the product Herz spaces and the product MorreyHerz spaces, respectively. We present a sufficient condition on the weight function that guarantees weighted multilinear Hardy operators to be bounded on the product Herz spaces. And the condition is necessary under certain assumptions. Finally, we extend the obtained results to the product Morrey-Herz spaces.
\end{abstract}

\section{Introduction}

Let $f$ be a nonnegative integral function on $\mathbb{R}^{+}$. The Hardy operator $H$ is defined by $H f(x)=(1 / x) \int_{0}^{x} f(t) d t, x>0$. For the operator $H$, Hardy et al. [1] proved that the inequality

$$
\|H f\|_{L^{p}} \leq \frac{p}{p-1}\|f\|_{L^{p}}
$$

holds, where $1<p<\infty$ and the constant $p /(p-1)$ is the best possible. Usually, we call (1) classical Hardy's inequality. With the development of analysis theory, many types of Hardy's inequalities have been discussed. For example, a quite number of papers dealt with the various generalizations, numerous variants, and applications of Hardy's inequalities in the past few years. On the detailed discussions of Hardy's inequalities, we choose to refer to $[2,3]$.

In 1984, Carton-Lebrun and Fosset [4] gave the definition of the weighted Hardy operator $H_{\omega}$ to be

$$
H_{\omega} f(x):=\int_{0}^{1} f(t x) \omega(t) d t, \quad x \in \mathbb{R}^{n},
$$

where $\omega:[0,1] \rightarrow[0, \infty)$ is a measurable function and $f$ is a complex-valued measurable function on $\mathbb{R}^{n}$. It is obvious that $H_{\omega}$ degenerates into the classical Hardy operator $H$ when $\omega \equiv 1, n=1$, and $f$ is defined on $\mathbb{R}^{+}$. In addition, we call the adjoint operator of $H_{\omega}$ the weighted Cesàro average $G_{\omega} f$. And the definition of $G_{\omega} f$ is

$$
\left(G_{\omega} f\right)(x)=\int_{0}^{1} f\left(\frac{x}{t}\right) t^{-n} \omega(t) d t
$$

When $\omega \equiv 1$ and $n=1, G_{\omega}$ becomes the classical Cesàro operator $G$

$$
G f(x)= \begin{cases}\int_{x}^{\infty} \frac{f(y)}{y} d y, & x>0, \\ -\int_{-\infty}^{x} \frac{f(y)}{y} d y, & x<0 .\end{cases}
$$

It is easy to get that $H_{\omega}$ and $G_{\omega}$ satisfy

$$
\int_{\mathbb{R}^{n}}\left(H_{\omega} f\right)(x) g(x) d x=\int_{\mathbb{R}^{n}} f(x)\left(G_{\omega} g\right)(x) d x,
$$

when $f \in L^{p}\left(\mathbb{R}^{n}\right), g \in L^{q}\left(\mathbb{R}^{n}\right), 1<p<\infty, 1 / p+1 / q=1$. This means that $H_{\omega}$ and $G_{\omega}$ satisfy the commutative rule $H_{\omega} G_{\omega}=G_{\omega} H_{\omega}$.

Under certain conditions on $\omega$, Carton-Lebrun and Fosset [4] proved that $H_{\omega}$ maps $L^{p}\left(\mathbb{R}^{n}\right)$ into itself for $1<p<\infty$. They also pointed out that the operator $H_{\omega}$ commutes with the Hilbert transform when $n=1$ and with certain CalderónZygmund singular integral operators including the Riesz 
transform when $n \geq 2$. Refer to Xiao [5] for the further extension of the results above; see also $[6,7]$.

Since Herz space is a natural generalization of weighted Lebesgue spaces with power weights, researchers are also interested in studying the boundedness of $H_{\omega}$ on Herz spaces. To make the description more clear below, we review the definition of the Herz spaces now. In the following definitions, $\chi_{k}=\chi_{C_{k}}, C_{k}=B_{k} \backslash B_{k-1}$, and $B_{k}=\left\{x \in \mathbb{R}^{n}\right.$ : $\left.|x| \leq 2^{k}\right\}$, for $k \in \mathbb{Z}, \widetilde{C}_{k}=C_{k}, \widetilde{C}_{0}=B_{0}$, and $\widetilde{\chi}_{k}=\chi_{C_{k}}$, for $k \in \mathbb{N}$, and $\chi_{E}$ is the characteristic function of a set $E$.

Definition 1 (see [8]). Let $\alpha \in \mathbb{R}, 0<p \leq \infty, 0<q<\infty$.

(1) The homogeneous Herz spaces $\dot{K}_{q}^{\alpha, p}\left(\mathbb{R}^{n}\right)$ is defined by

$$
\dot{K}_{q}^{\alpha, p}\left(\mathbb{R}^{n}\right)=\left\{f \in L_{\text {loc }}^{q}\left(\mathbb{R}^{n} \backslash\{0\}\right):\|f\|_{\dot{K}_{q}^{\alpha, p}\left(\mathbb{R}^{n}\right)}<\infty\right\},
$$

where

$$
\|f\|_{\dot{K}_{q}^{\alpha, p}\left(\mathbb{R}^{n}\right)}=\left\{\sum_{k=-\infty}^{\infty} 2^{k \alpha p}\left\|f \chi_{k}\right\|_{L^{q}\left(\mathbb{R}^{n}\right)}^{p}\right\}^{1 / p} .
$$

(2) The inhomogenous Herz spaces $K_{q}^{\alpha, p}\left(\mathbb{R}^{n}\right)$ is defined by

$$
K_{q}^{\alpha, p}\left(\mathbb{R}^{n}\right)=\left\{f \in L_{\text {loc }}^{q}\left(\mathbb{R}^{n}\right):\|f\|_{K_{q}^{\alpha, p}\left(\mathbb{R}^{n}\right)}<\infty\right\}
$$

where

$$
\|f\|_{K_{q}^{\alpha, p}\left(\mathbb{R}^{n}\right)}=\left\{\sum_{k=0}^{\infty} 2^{k \alpha p}\left\|f \tilde{\chi}_{k}\right\|_{L^{q}\left(\mathbb{R}^{n}\right)}^{p}\right\}^{1 / p},
$$

with usual modifications made when $p=\infty$ or $q=\infty$.

In [9], Wu gave the definition of Morrey-Herz spaces.

Definition 2. Let $\alpha \in \mathbb{R}, 0<p \leq \infty, 0<q<\infty$, and $\lambda \geq 0$.

(1) The homogeneous Morrey-Herz space $M \dot{K}_{p, q}^{\alpha, \lambda}\left(\mathbb{R}^{n}\right)$ is defined by

$$
M \dot{K}_{p, q}^{\alpha, \lambda}\left(\mathbb{R}^{n}\right):=\left\{f \in L_{\text {loc }}^{q}\left(\mathbb{R}^{n} \backslash\{0\}\right):\|f\|_{M \dot{K}_{p, q}^{\alpha, \lambda}\left(\mathbb{R}^{n}\right)}<\infty\right\},
$$

where

$$
\|f\|_{M \dot{K}_{p, q}^{\alpha, \lambda}\left(\mathbb{R}^{n}\right)}:=\sup _{k_{0} \in \mathbb{Z}} 2^{-k_{0} \lambda}\left\{\sum_{k=-\infty}^{k_{0}} 2^{k \alpha p}\left\|f \chi_{k}\right\|_{L^{q}\left(\mathbb{R}^{n}\right)}^{p}\right\}^{1 / p} .
$$

(2) The inhomogeneous Morrey-Herz space $M K_{p, q}^{\alpha, \lambda}\left(\mathbb{R}^{n}\right)$ is defined by

$$
M K_{p, q}^{\alpha, \lambda}\left(\mathbb{R}^{n}\right):=\left\{f \in L_{\text {loc }}^{q}\left(\mathbb{R}^{n}\right):\|f\|_{M K_{p, q}^{\alpha, \lambda}\left(\mathbb{R}^{n}\right)}<\infty\right\},
$$

where

$$
\|f\|_{M K_{p, q}^{\alpha, \lambda}\left(\mathbb{R}^{n}\right)}:=\sup _{k_{0} \in \mathbb{N}} 2^{-k_{0} \lambda}\left\{\sum_{k=0}^{k_{0}} 2^{k \alpha p}\left\|f \tilde{\chi}_{k}\right\|_{L^{q}\left(\mathbb{R}^{n}\right)}^{p}\right\}^{1 / p},
$$

with usual modifications made when $p=\infty$ or $q=\infty$.
From the above definitions, it is not difficult to note that $\dot{K}_{p}^{0, p}\left(\mathbb{R}^{n}\right)=K_{p}^{0, p}\left(\mathbb{R}^{n}\right)=L^{p}\left(\mathbb{R}^{n}\right), \dot{K}_{p}^{\alpha / p, p}\left(\mathbb{R}^{n}\right)=K_{p}^{\alpha / p, p}\left(\mathbb{R}^{n}\right)=$ $L^{p}\left(|x|^{\alpha} d x\right)$ for all $0<p \leq \infty$ and $\alpha \in \mathbb{R}$. Moreover, the homogenous Herz spaces $\dot{K}_{q}^{\alpha, p}\left(\mathbb{R}^{n}\right)$, the homogenous Morrey-Herz spaces $M \dot{K}_{p, q}^{\alpha, \lambda}\left(\mathbb{R}^{n}\right)$, and the Morrey spaces $M^{q, \lambda}\left(\mathbb{R}^{n}\right)$ (see $\left.[10,11]\right)$ satisfy $M \dot{K}_{p, q}^{\alpha, 0}\left(\mathbb{R}^{n}\right)=\dot{K}_{q}^{\alpha, p}\left(\mathbb{R}^{n}\right)$, $M^{q, \lambda}\left(\mathbb{R}^{n}\right) \subset M \dot{K}_{q, q}^{0, \lambda}\left(\mathbb{R}^{n}\right)$. In [12], Liu and Fu discussed the boundedness of the weighted Hardy operator $H_{\omega}$ on the Herz space $\dot{K}_{q}^{\alpha, p}\left(\mathbb{R}^{n}\right)$. Conditions on the weighted function $\omega$ were presented to guarantee that $H_{\omega}$ is bounded on $\dot{K}_{q}^{\alpha, p}\left(\mathbb{R}^{n}\right)$. They also estimated the corresponding operator norm. And in [13], $\mathrm{Fu}$ and $\mathrm{Lu}$ further extended the results of [12] to the MorreyHerz space $M \dot{K}_{p, q}^{\alpha, \lambda}\left(\mathbb{R}^{n}\right)$.

In the past few years, the properties of multilinear operators have also been extensively studied by researchers. There are two reasons for this. First, the multilinear operators are the generalization of the linear ones, and its study makes the research contents of analysis theory more rich. Second, the multilinear operators naturally appear in analysis. The study of multilinear operators is traced to the multilinear singular integral operator theory (see [14]). For more detailed studies on multilinear operators, the readers refer to [1518 ] and the references therein. Recently, we have studied the boundedness of weighted multilinear Hardy operators $\mathscr{H}_{\omega}^{m}$ on the product of Lebesgue spaces and central Morrey spaces in [19]. Based on these results and inspired by the results of $[12,13]$, this paper further concerns the boundedness of $\mathscr{H}_{\omega}^{m}$ on the product Herz spaces and the product Morrey-Herz spaces. We first recall the definition of $\mathscr{H}_{\omega}^{m}$.

Definition 3. Let $m \in \mathbb{N}, m \geq 2$, and

$$
\omega: \overbrace{[0,1] \times[0,1] \times \cdots \times[0,1]}^{m \text { times }} \longrightarrow[0, \infty)
$$

be an integrable function. The weighted multilinear Hardy operator $\mathscr{H}_{\omega}^{m}$ is defined by

$$
\mathscr{H}_{\omega}^{m}(\vec{f})(x):=\int_{0<t_{1}, t_{2}, \ldots, t_{m}<1}\left(\prod_{i=1}^{m} f_{i}\left(t_{i} x\right)\right) \omega(\vec{t}) d \vec{t}
$$

$$
x \in \mathbb{R}^{n},
$$

where $\vec{f}:=\left(f_{1}, \ldots, f_{m}\right), \omega(\vec{t}):=\omega\left(t_{1}, t_{2}, \ldots, t_{m}\right), d \vec{t}:=$ $d t_{1} \cdots d t_{m}$, and $f_{i}(i=1, \ldots, m)$ are complex-valued measurable functions on $\mathbb{R}^{n}$. When $m=2, \mathscr{H}_{\omega}^{m}$ is referred to as bilinear.

In accordance with the case of $H_{\omega}$, we also recall the definition of the weighted multilinear Cesàro operator $\mathscr{G}_{\omega}$ that is the adjoint operator of $\mathscr{H}_{\omega}^{m}$. 
Definition 4. Let $m \in \mathbb{N}, m \geq 2$, and $\omega:[0,1] \times[0,1]^{m-1} \rightarrow$ $[0, \infty)$ be an integrable function. The weighted multilinear Cesàro operator $\mathscr{G}_{\omega}^{m}$ is defined by

$$
\begin{array}{r}
\mathscr{G}_{\omega}^{m}(\vec{f})(x)=\int_{0<t_{1}, t_{2}, \ldots, t_{m}<1}\left(\prod_{i=1}^{m} f_{i}\left(\frac{x}{t_{i}}\right)\left(t_{i}\right)^{-n}\right) \omega(\vec{t}) d \vec{t}, \\
x \in \mathbb{R}^{n},
\end{array}
$$

where $f_{i}$ are measurable complex-valued functions on $\mathbb{R}^{n}, 1 \leq i \leq m$.

Note that $\mathscr{H}_{\omega}^{m}$ and $\mathscr{G}_{\omega}^{m}$ do not satisfy the following commutative rule:

$$
\int_{\mathbb{R}^{n}}\left(\mathscr{H}_{\omega}^{m} f\right)(x) g(x) d x=\int_{\mathbb{R}^{n}} f(x)\left(\mathscr{G}_{\omega}^{m} g\right)(x) d x .
$$

This is different from the case of $H_{\omega}$ and $G_{\omega}$.

The paper is organized as follows. In Section 2, we present the estimate of the boundedness of $\mathscr{H}_{\omega}^{m}$ on the product Herz spaces. In Section 3, we give the estimates of the boundedness of $\mathscr{H}_{\omega}^{m}$ on the product Morrey-Herz spaces.

\section{Boundedness of $\mathscr{H}_{\omega}^{m}$ on the Product of Herz Spaces}

We give the first main result of this paper.

Theorem 5. Let $\alpha, \alpha_{1}, \ldots, \alpha_{2} \in \mathbb{R}, 1<p, p_{i}, q, q_{i}<\infty$ and $\alpha_{1}+\cdots \alpha_{m}=\alpha, 1 / q_{1}+\cdots 1 / q_{m}=1 / q, 1 / p_{1}+\cdots 1 / p_{m}=$ $1 / p, i=1,2, \ldots, m$. Then $\mathscr{H}_{\omega}^{m}$ is bounded from $\dot{K}_{q_{1}}^{\alpha_{1}, p_{1}} \times \cdots \times$ $\dot{K}_{q_{m}}^{\alpha_{m}, p_{m}}$ to $\dot{K}_{q}^{\alpha, p}$ if

$$
\int_{0<t_{1}, \ldots, t_{m}<1}\left(\prod_{i=1}^{m} t_{i}^{-\left(\alpha_{i}+n / q_{i}\right)}\right) \omega(\vec{t}) d \vec{t}<\infty .
$$

Conversely, if $\alpha_{1}=\cdots=\alpha_{m}=(1 / m) \alpha, q_{i}=m q, p_{i}=m p$, $i=1,2, \ldots, m$, and $\mathscr{H}_{\omega}^{m}$ is bounded from $\dot{K}_{q_{1}}^{\alpha_{1}, p_{1}} \times \cdots \times \dot{K}_{q_{m}}^{\alpha_{m}}, p_{m}$ to $\dot{K}_{q}^{\alpha, p}$, then (18) holds. Moreover, in this case, one has

$$
\begin{aligned}
& \left\|\mathscr{H}_{\omega}^{m}\right\|_{\dot{K}_{q_{1}}^{\alpha_{1}, p_{1}} \times \cdots \times \dot{K}_{q_{m}}^{\alpha_{m}, p_{m}} \rightarrow \dot{K}_{q}^{\alpha, p}} \\
& \quad \simeq \int_{0<t_{1}, \ldots, t_{m}<1}\left(\prod_{i=1}^{m} t_{i}^{-\left(\alpha_{i}+n / q_{i}\right)}\right) \omega(\vec{t}) d \vec{t} .
\end{aligned}
$$
$\mathscr{G}_{\omega}^{m}$.

Similarly, we have the following result for the operator

Theorem 6. Let $\alpha, \alpha_{1}, \ldots, \alpha_{2} \in \mathbb{R}, 1<p, p_{i}, q, q_{i}<\infty$, and $\alpha_{1}+\cdots \alpha_{m}=\alpha, 1 / q_{1}+\cdots 1 / q_{m}=1 / q, 1 / p_{1}+\cdots 1 / p_{m}=1 / p$, $i=1,2, \ldots, m$. If $\omega$ satisfies

$$
\int_{0<t_{1}, \ldots, t_{m}<1}\left(\prod_{i=1}^{m} t_{i}^{\alpha_{i}-n\left(1-1 / q_{i}\right)}\right) \omega(\vec{t}) d \vec{t}<\infty,
$$

then $\mathscr{G}_{\omega}^{m}$ is bounded from $\dot{K}_{q_{1}}^{\alpha_{1}, p_{1}} \times \cdots \times \dot{K}_{q_{m}}^{\alpha_{m}}, p_{m}$ to $\dot{K}_{q}^{\alpha, p}$.
Conversely, if $\alpha_{1}=\cdots=\alpha_{m}=(1 / m) \alpha, q_{i}=m q, p_{i}=m p$, $i=1,2, \ldots, m$, and $\mathscr{G}_{\omega}^{m}$ is bounded from $\dot{K}_{q_{1}}^{\alpha_{1}, p_{1}} \times \cdots \times \dot{K}_{q_{m}}^{\alpha_{m}, p_{m}}$ to $\dot{K}_{q}^{\alpha, p}$, then (20) holds. Moreover, in this case, one has

$$
\begin{aligned}
& \left\|\mathscr{G}_{\omega}^{m}\right\|_{\dot{K}_{q_{1}}^{\alpha_{1}, p_{1}} \times \cdots \times \dot{K}_{q_{m}}^{\alpha_{m}, p_{m}}} \rightarrow \dot{K}_{q}^{\alpha, p} \\
& \quad \simeq \int_{0<t_{1}, \ldots, t_{m}<1}\left(\prod_{i=1}^{m} t_{i}^{\alpha_{i}-n\left(1-1 / q_{i}\right)}\right) \omega(\vec{t}) d \vec{t} .
\end{aligned}
$$

Since the proofs of Theorems 5 and 6 are similar, we just give the proof of Theorem 5 .

Proof of Theorem 5. In order to simplify the proof, we only consider the case of $m=2$. Actually, a similar procedure works for all $m \in \mathbb{N}$.

Since $1 / q_{1}+1 / q_{2}=1 / q$, by Hölder and Minkowski inequality, we have

$$
\begin{aligned}
& \left\|\mathscr{H}_{\omega}^{2}\left(f_{1}, f_{2}\right) \chi_{k}\right\|_{L^{q}\left(\mathbb{R}^{n}\right)} \\
& \left.=\iint_{C_{k}}\left|\iint_{0}^{1} f_{1}\left(t_{1} x\right) f\left(t_{2} x\right) \omega\left(t_{1}, t_{2}\right) d t_{1} d t_{2}\right|^{q}\right)^{1 / q} \\
& \leq \iint_{0}^{1}\left(\int_{C_{k}}\left|f_{1}\left(t_{1} x\right) f\left(t_{2} x\right)\right|^{q}\right)^{1 / q} \omega\left(t_{1}, t_{2}\right) d t_{1} d t_{2} \\
& \leq \iint_{0}^{1}\left(\int_{C_{k}}\left|f_{1}\left(t_{1} x\right)\right|^{q_{1}} d x\right)^{1 / q_{1}} \\
& \quad \times\left(\int_{C_{k}}\left|f_{2}\left(t_{2} x\right)\right|^{q_{2}} d x\right)^{1 / q_{2}} \omega\left(t_{1}, t_{2}\right) d t_{1} d t_{2} .
\end{aligned}
$$

For the arbitrary $t_{1}, t_{2} \in(0,1)$, we can find $m, l \in \mathscr{Z}$, such that $2^{m-1}<t_{1} \leq 2^{m}$ and $2^{l-1}<t_{2} \leq 2^{l}$. By Minkowski inequality, we have

$$
\begin{aligned}
& \iint_{0}^{1}\left(\int_{C_{k}}\left|f_{1}\left(t_{1} x\right)\right|^{q_{1}} d x\right)^{1 / q_{1}} \\
& \quad \times\left(\int_{C_{k}}\left|f_{2}\left(t_{2} x\right)\right|^{q_{2}} d x\right)^{1 / q_{2}} \omega\left(t_{1}, t_{2}\right) d t_{1} d t_{2} \\
& \leq \iint_{0}^{1}\left(\int_{t_{1} C_{k}}\left|f_{1}(x)\right|^{q_{1}} d x\right)^{1 / q_{1}} \\
& \quad \times\left(\int_{t_{2} C_{k}}\left|f_{2}(x)\right|^{q_{2}} d x\right)^{1 / q_{2}} t_{1}^{-n / q_{1}} t_{2}^{-n / q_{2}} \omega\left(t_{1}, t_{2}\right) d t_{1} d t_{2} \\
& \leq \iint_{0}^{1}\left(\int_{2^{k+m-2}<|x| \leq 2^{k+m}}\left|f_{1}(x)\right|^{q_{1}} d x\right)^{1 / q_{1}} \\
& \quad \times\left(\int_{2^{k+l-2}<|x| \leq 2^{k+l}}\left|f_{2}(x)\right|^{q_{2}} d x\right)^{1 / q_{2}} \\
& \quad \times t_{1}^{-n / q_{1}} t_{2}^{-n / q_{2}} \omega\left(t_{1}, t_{2}\right) d t_{1} d t_{2}
\end{aligned}
$$




$$
\begin{aligned}
\leq \iint_{0}^{1} & \left(\left\|f_{1} \chi_{k+m-1}\right\|_{L^{q_{1}}}+\left\|f_{1} \chi_{k+m}\right\|_{L^{q_{1}}}\right) \\
& \times\left(\left\|f_{2} \chi_{k+l-1}\right\|_{L^{q_{2}}}+\left\|f_{2} \chi_{k+l}\right\|_{L^{q_{2}}}\right) t_{1}^{-n / q_{1}} \\
& \times t_{2}^{-n / q_{2}} \omega\left(t_{1}, t_{2}\right) d t_{1} d t_{2} .
\end{aligned}
$$

For $1 / p_{1}+1 / p_{2}=1 / p$ and $\alpha_{1}+\alpha_{2}=\alpha$, Hölder inequality and Minkowski inequality give

$$
\begin{aligned}
& \left\|\mathscr{H}_{\omega}^{2}\left(f_{1}, f_{2}\right)\right\|_{\dot{K}_{q}^{\alpha, p}} \\
& =\left(\sum_{k=-\infty}^{\infty} 2^{k \alpha p}\left\|\mathscr{H}_{\omega}^{2}\left(f_{1}, f_{2}\right) \chi_{k}\right\|_{L^{q}\left(\mathbb{R}^{n}\right)}^{p}\right)^{1 / p} \\
& \leq\left(\sum _ { k = - \infty } ^ { \infty } 2 ^ { k \alpha p } \left(\iint_{0}^{1}\left(\left\|f_{1} \chi_{k+m-1}\right\|_{L^{q_{1}}}+\left\|f_{1} \chi_{k+m}\right\|_{L^{q_{1}}}\right)\right.\right. \\
& \times\left(\left\|f_{2} \chi_{k+l-1}\right\|_{L^{q_{2}}}+\left\|f_{2} \chi_{k+l}\right\|_{L^{q_{2}}}\right) \\
& \left.\left.\times t_{1}^{-n / q_{1}} t_{2}^{-n / q_{2}} \omega\left(t_{1}, t_{2}\right) d t_{1} d t_{2}\right)^{p}\right)^{1 / p} \\
& \leq \iint_{0}^{1}\left(\sum_{k=-\infty}^{\infty} 2^{k \alpha p}\left(\left\|f_{1} \chi_{k+m-1}\right\|_{L^{q_{1}}}+\left\|f_{1} \chi_{k+m}\right\|_{L^{q_{1}}}\right)^{p}\right. \\
& \left.\times\left(\left\|f_{2} \chi_{k+l-1}\right\|_{L^{q_{2}}}+\left\|f_{2} \chi_{k+l}\right\|_{L^{q_{2}}}\right)^{p}\right)^{1 / p} \\
& \times t_{1}^{-n / q_{1}} t_{2}^{-n / q_{2}} \omega\left(t_{1}, t_{2}\right) d t_{1} d t_{2} \\
& \leq \iint_{0}^{1}\left(\sum _ { k = - \infty } ^ { \infty } 2 ^ { k \alpha _ { 1 } p _ { 1 } } \left(\left\|f_{1} \chi_{k+m-1}\right\|_{L^{q_{1}}}\right.\right. \\
& \left.\left.+\left\|f_{1} \chi_{k+m}\right\|_{L^{q_{1}}}\right)^{p_{1}}\right)^{1 / p_{1}} \\
& \times\left(\sum_{k=-\infty}^{\infty} 2^{k \alpha_{2} p_{2}}\left(\left\|f_{2} \chi_{k+l-1}\right\|_{L^{q_{2}}}+\left\|f_{2} \chi_{k+l}\right\|_{L^{q_{2}}}\right)^{p_{2}}\right)^{1 / p_{2}} \\
& \times t_{1}^{-n / q_{1}} t_{2}^{-n / q_{2}} \omega\left(t_{1}, t_{2}\right) d t_{1} d t_{2} \\
& \leq \iint_{0}^{1}\left\{\left(\sum_{k=-\infty}^{\infty} 2^{k \alpha_{1} p_{1}}\left\|f_{1} \chi_{k+m-1}\right\|_{L^{q_{1}}}^{p_{1}}\right)^{1 / p_{1}}\right. \\
& \left.+\left(\sum_{k=-\infty}^{\infty} 2^{k \alpha_{1} p_{1}}\left\|f_{1} \chi_{k+m}\right\|_{L^{q_{1}}}^{p_{1}}\right)^{1 / p_{1}}\right\} \\
& \times\left\{\left(\sum_{k=-\infty}^{\infty} 2^{k \alpha_{2} p_{2}}\left\|f_{2} \chi_{k+l-1}\right\|_{L^{q_{2}}}^{p_{2}}\right)^{1 / p_{2}}\right.
\end{aligned}
$$

$$
\begin{aligned}
& \left.+\left(\sum_{k=-\infty}^{\infty} 2^{k \alpha_{2} p_{2}}\left\|f_{2} \chi_{k+1}\right\|_{L^{q_{2}}}^{p_{2}}\right)^{1 / p_{2}}\right\} \\
& \times t_{1}^{-n / q_{1}} t_{2}^{-n / q_{2}} \omega\left(t_{1}, t_{2}\right) d t_{1} d t_{2} \\
& \leq\left\|f_{1}\right\|_{\dot{K}_{q_{1}}^{\alpha_{1}, p_{1}}}\left\|f_{2}\right\|_{\dot{K}_{q_{2}}^{\alpha_{2}, p_{2}}} \\
& \times \iint_{0}^{1}\left(2^{-(m-1) \alpha_{1}}+2^{-m \alpha_{1}}\right)\left(2^{-(l-1) \alpha_{2}}+2^{-l \alpha_{2}}\right) \\
& \times t_{1}^{-n / q_{1}} t_{2}^{-n / q_{2}} \omega\left(t_{1}, t_{2}\right) d t_{1} d t_{2} \\
& \leq\left\|f_{1}\right\|_{\dot{K}_{q_{1}}^{\alpha_{1}, p_{1}}}\left\|f_{2}\right\|_{\dot{K}_{q_{2}}^{\alpha_{2}, p_{2}}} \\
& \times \iint_{0}^{1} t_{1}^{-\left(\alpha_{1}+n / q_{1}\right)} t_{2}^{-\left(\alpha_{2}+n / q_{2}\right)} \omega\left(t_{1}, t_{2}\right) d t_{1} d t_{2} .
\end{aligned}
$$

From the above inequality, we have that the first conclusion in Theorem 5 holds.

On the other hand, we suppose that $\mathscr{H}_{\omega}^{2}$ is bounded from $\dot{K}_{q_{1}}^{\alpha_{1}, p_{1}} \times \dot{K}_{q_{2}}^{\alpha_{2}, p_{2}}$ to $\dot{K}_{q}^{\alpha, p}$ and $\mathscr{H}_{\omega}^{2}$ has the operator norm $\left\|\mathscr{H}_{\omega}^{2}\right\|_{\dot{K}_{q_{1}}^{\alpha_{1}, p_{1}} \times \dot{K}_{q_{2}}^{\alpha_{2}, p_{2}} \rightarrow \dot{K}_{q}^{\alpha, p} . \text { For any } 0<\varepsilon<1 \text {, let }}$

$$
\begin{aligned}
& f_{1}(x)= \begin{cases}0, & |x| \leq 1, \\
|x|^{-\alpha_{1}-n / q_{1}-\varepsilon}, & |x|>1,\end{cases} \\
& f_{2}(x)= \begin{cases}0, & |x| \leq 1, \\
|x|^{-\alpha_{2}-n / q_{2}-\varepsilon}, & |x|>1 .\end{cases}
\end{aligned}
$$

Obviously, $\left\|f_{1} \chi_{k}\right\|_{L^{q_{1}\left(\mathbb{R}^{n}\right)}}=\left\|f_{2} \chi_{k}\right\|_{L^{q_{2}\left(\mathbb{R}^{n}\right)}}=0$, when $k=$ $0,-1,-2, \ldots$. So, for any positive integer $k$, it is easy to get that

$$
\begin{aligned}
\left\|f_{1} \chi_{k}\right\|_{L^{q_{1}\left(\mathbb{R}^{n}\right)}} & =\left(\int_{2^{k-1} \leq|x|<2^{k}}|x|^{\left(-\alpha_{1}-n / q_{1}-\varepsilon\right) q_{1}} d x\right)^{1 / q_{1}} \\
& =2^{-\left(\alpha_{1}+\varepsilon\right) k}\left|\frac{S_{n}\left(2^{\left(\alpha_{1}+\varepsilon\right) q_{1}}-1\right)}{\left(\alpha_{1}+\varepsilon\right) q_{1}}\right|^{1 / q_{1}}
\end{aligned}
$$

where $S_{n}=n \pi^{n / 2} / \Gamma(1+n / 2)$. A simple computation gives

$$
\begin{aligned}
& \left\|f_{1}\right\|_{\dot{K}_{q_{1}}^{\alpha_{1}, p_{1}}\left(\mathbb{R}^{n}\right)} \\
& \quad=2^{-\varepsilon}\left(\frac{1}{1-2^{-\varepsilon p_{1}}}\right)^{1 / p_{1}}\left|\frac{S_{n}\left(2^{\left(\alpha_{1}+\varepsilon\right) q_{1}}-1\right)}{\left(\alpha_{1}+\varepsilon\right) q_{1}}\right|^{1 / q_{1}} .
\end{aligned}
$$

Similarly, we obtain

$$
\begin{aligned}
& \left\|f_{2}\right\|_{\dot{K}_{q_{2}}^{\alpha_{2}, p_{2}}\left(\mathbb{R}^{n}\right)} \\
& \quad=2^{-\varepsilon}\left(\frac{1}{1-2^{-\varepsilon p_{2}}}\right)^{1 / p_{2}}\left|\frac{S_{n}\left(2^{\left(\alpha_{2}+\varepsilon\right) q_{2}}-1\right)}{\left(\alpha_{2}+\varepsilon\right) q_{2}}\right|^{1 / q_{2}} .
\end{aligned}
$$


When $|x| \leq 1$ and $t \in[0,1]$, we have $|t x| \leq 1$. So, $\mathscr{H}_{\omega}^{2}\left(f_{1}, f_{2}\right)(x)=0$ in this case based on (25). When $|x|>1$, we get

$$
\begin{aligned}
& \mathscr{H}_{\omega}^{2}\left(f_{1}, f_{2}\right)(x) \\
&=\iint_{1 /|x|}^{1} f_{1}\left(t_{1} x\right) f_{2}\left(t_{2} x\right) \omega\left(t_{1}, t_{2}\right) d t_{1} d t_{2} \\
&=|x|^{-(\alpha+(n / q)+2 \varepsilon)} \iint_{1 /|x|}^{1} t_{1}^{-\alpha_{1}-n / q_{1}-\varepsilon} t_{2}^{-\alpha_{2}-n / q_{2}-\varepsilon} \\
& \times \omega\left(t_{1}, t_{2}\right) d t_{1} d t_{2} .
\end{aligned}
$$

Let $\delta=\varepsilon^{-1}>1$. It is easy to find a positive integer $l$ such that $2^{l-1} \leq \delta<2^{l}$. Thus, we have

$$
\begin{aligned}
& \left\|\mathscr{H}_{\omega}^{2}\left(f_{1}, f_{2}\right)\right\|_{\dot{K}_{q}^{\alpha, p}}^{p} \\
& =\sum_{k=1}^{\infty} 2^{k \alpha p}\left\|\mathscr{H}_{\omega}^{2}\left(f_{1}, f_{2}\right) \chi_{k}(x)\right\|_{L^{q}\left(\mathbb{R}^{n}\right)}^{p} \\
& =\sum_{k=1}^{\infty} 2^{k \alpha p}\left\{\int _ { | x | > 1 } \left(|x|^{-(\alpha+n / q+2 \varepsilon)} \chi_{k}(x)\right.\right. \\
& \quad \times \iint_{1 /|x|}^{1} t_{1}^{-\alpha_{1}-n / q_{1}-\varepsilon} t_{2}^{-\alpha_{2}-n / q_{2}-\varepsilon} \\
& \geq \sum_{k=1}^{\infty} 2^{k \alpha p}\left(\int_{|x|>\delta}^{|x|^{-(\alpha+n / q+2 \varepsilon) q}} \chi_{k}(x) d x\right)^{p / q} \\
& \left.\left.\quad \times\left(\iint_{1 / \delta}^{1} t_{1}^{-\alpha_{1}-n / q_{1}-\varepsilon} t_{2}^{-\alpha_{2}-n / q_{2}-\varepsilon} \omega\left(t_{1}, t_{2}\right) d t_{1} d t_{2}\right)^{p} d t_{1} d t_{2}\right)^{q} d x\right\}^{p / q} \\
& \geq S_{n}^{p / q} \sum_{k=l+1}^{\infty} 2^{k \alpha p}\left(\int_{2^{k-1}}^{2^{k}} r^{-(\alpha+2 \varepsilon) q-1} d r\right)^{p / q} \\
& \quad \times\left(\iint_{1 / \delta}^{1} t_{1}^{-\alpha_{1}-n / q_{1}-\varepsilon} t_{2}^{-\alpha_{2}-n / q_{2}-\varepsilon} \omega\left(t_{1}, t_{2}\right) d t_{1} d t_{2}\right)^{p} \\
& \quad \times\left(\iint_{1 / \delta}^{1} t_{1}^{-\alpha_{1}-n / q_{1}-\varepsilon} t_{2}^{-\alpha_{2}-n / q_{2}-\varepsilon} \omega\left(t_{1}, t_{2}\right) d t_{1} d t_{2}\right)^{p} \\
& \quad S_{n}^{p / q} \sum_{k=l+1}^{\infty} 2^{-2 \varepsilon p k}\left|\frac{\left(2^{(\alpha+2 \varepsilon) q}-1\right)}{(\alpha+2 \varepsilon) q}\right|^{p / q} \\
& \quad \times\left(2_{1}^{-2 \varepsilon(l+1) p} \mid \frac{\left(2^{(\alpha+2 \varepsilon) q}-1\right)}{\left(\alpha+q_{1}-\varepsilon\right.} t_{2}^{-\alpha_{2}-n / q_{2}-\varepsilon} \omega\left(t_{1}, t_{2}\right) d t_{1} d t_{2}\right)^{p / q}
\end{aligned}
$$

Since $q_{1}=q_{2}=2 q, \alpha_{1}=\alpha_{2}=(1 / 2) \alpha$ and $1 / p=1 / p_{1}+1 / p_{2}$, $p_{1}=p_{2}=2 p$, we have

$$
\begin{aligned}
& \left\|\mathscr{H}_{\omega}^{2}\left(f_{1}, f_{2}\right)\right\|_{\dot{K}_{q}^{\alpha, p}} \\
& \geq S_{n}^{1 / q_{2}} 2^{-2 \varepsilon(l+1)}\left(\frac{1}{1-2^{-2 \varepsilon p}}\right)^{1 / p}\left|\frac{\left(2^{(\alpha+2 \varepsilon) q}-1\right)}{(\alpha+2 \varepsilon) q}\right|^{1 / q} \\
& \quad \times \iint_{1 / \delta}^{1} t_{1}^{-\alpha_{1}-n / q_{1}-\varepsilon} t_{2}^{-\alpha_{2}-n / q_{2}-\varepsilon} \omega\left(t_{1}, t_{2}\right) d t_{1} d t_{2} \\
& \geq \varepsilon^{2 \varepsilon}\left\|f_{1}\right\|_{\dot{K}_{q_{1}}^{\alpha_{1}, p_{1}}}\left\|f_{2}\right\|_{\dot{K}_{q_{2}}^{\alpha_{2}, p_{2}}} \\
& \quad \times \iint_{\varepsilon}^{1} t_{1}^{-\alpha_{1}-n / q_{1}-\varepsilon} t_{2}^{-\alpha_{2}-n / q_{2}-\varepsilon} \omega\left(t_{1}, t_{2}\right) d t_{1} d t_{2} .
\end{aligned}
$$

This gives

$$
\begin{aligned}
& \left\|\mathscr{H}_{\omega}^{2}\left(f_{1}, f_{2}\right)\right\|_{\dot{K}_{q}^{\alpha, p}} \\
& \geq \varepsilon^{2 \varepsilon}\left\|f_{1}\right\|_{\dot{K}_{q_{1}}^{\alpha_{1}, p_{1}}}\left\|f_{2}\right\|_{\dot{K}_{q_{2}}^{\alpha_{2}, p_{2}}} \\
& \quad \times \iint_{\varepsilon}^{1} t_{1}^{-\alpha_{1}-n / q_{1}-\varepsilon} t_{2}^{-\alpha_{2}-n / q_{2}-\varepsilon} \omega\left(t_{1}, t_{2}\right) d t_{1} d t_{2} .
\end{aligned}
$$

Using the boundedness of $\mathscr{H}_{\omega}^{2}$ and its operator norm yields

$$
\begin{gathered}
\varepsilon^{2 \varepsilon} \iint_{\varepsilon}^{1} t_{1}^{-\alpha_{1}-n / q_{1}-\varepsilon} t_{2}^{-\alpha_{2}-n / q_{2}-\varepsilon} \omega\left(t_{1}, t_{2}\right) d t_{1} d t_{2} \\
\quad \leq\left\|\mathscr{H}_{\omega}^{2}\right\|_{\dot{K}_{q_{1}}^{\alpha_{1}, p_{1}} \times \dot{K}_{q_{2}}^{\alpha_{2}, p_{2}} \rightarrow \dot{K}_{q}^{\alpha, p p} .}
\end{gathered}
$$

Letting $\varepsilon \rightarrow 0^{+}$in (33) gives

$$
\begin{gathered}
\iint_{0}^{1} t_{1}^{-\alpha_{1}-n / q_{1}} t_{2}^{-\alpha_{2}-n / q_{2}} \omega\left(t_{1}, t_{2}\right) d t_{1} d t_{2} \\
\leq\left\|\mathscr{H}_{\omega}^{2}\right\|_{\dot{K}_{q_{1}}^{\alpha_{1}, p_{1}} \times \dot{K}_{q_{2}}^{\alpha_{2}, p_{2}} \rightarrow \dot{K}_{q}^{\alpha, p} .}^{\alpha_{1} .}
\end{gathered}
$$

Thus, (18) holds.

Since $\dot{K}_{p}^{0, p}\left(\mathbb{R}^{n}\right)=K_{p}^{0, p}\left(\mathbb{R}^{n}\right)=L^{p}\left(\mathbb{R}^{n}\right)$ and $\dot{K}_{p}^{\alpha / p, p}\left(\mathbb{R}^{n}\right)=$ $K_{p}^{\alpha / p, p}\left(\mathbb{R}^{n}\right)=L^{p}\left(|x|^{\alpha} d x\right)$ for all $0<p \leq \infty$, we deduce the following corollaries from Theorems 5 and 6 .

Corollary 7. Let $\alpha, \alpha_{1}, \ldots, \alpha_{2} \in \mathbb{R}, 1<p, p_{i}<\infty$ and $\alpha_{1}+$ $\cdots \alpha_{m}=\alpha, 1 / p_{1}+\cdots 1 / p_{m}=1 / p, i=1,2, \ldots, m$. Then $\mathscr{H}_{\omega}^{m}$ is bounded from $L^{p_{1}}\left(|x|^{\alpha_{1}} d x\right) \times \cdots \times L^{p_{m}}\left(|x|^{\alpha_{m}} d x\right)$ to $L^{p}\left(|x|^{\alpha} d x\right)$ if

$$
\int_{0<t_{1}, \ldots, t_{m}<1}\left(\prod_{i=1}^{m} t_{i}^{-\left(\alpha_{i}+n\right) / p_{i}}\right) \omega(\vec{t}) d \vec{t}<\infty .
$$

Conversely, if $\alpha_{1}=\cdots=\alpha_{m}=(1 / m) \alpha, p_{i}=m p, i=$ $1,2, \ldots, m$, and $\mathscr{H}_{\omega}^{m}$ is bounded from $L^{p_{1}}\left(|x|^{\alpha_{1}} d x\right) \times \cdots \times$ 
$L^{p_{m}}\left(|x|^{\alpha_{m}} d x\right)$ to $L^{p}\left(|x|^{\alpha} d x\right)$, then (35) holds. Moreover, in this case, one has

$$
\begin{aligned}
& \left\|\mathscr{H}_{\omega}^{m}\right\|_{L^{p_{1}}\left(|x|^{\alpha_{1}} d x\right) \times \cdots \times L^{p_{m}}\left(|x|^{\alpha_{m}} d x\right) \rightarrow L^{p}\left(|x|^{\alpha} d x\right)} \\
& \quad \int_{0<t_{1}, \ldots, t_{m}<1}\left(\prod_{i=1}^{m} t_{i}^{-\left(\alpha_{i}+n\right) / p_{i}}\right) \omega(\vec{t}) d \vec{t} .
\end{aligned}
$$

Corollary 8. Let $\alpha, \alpha_{1}, \ldots, \alpha_{2} \in \mathbb{R}, 1<p, p_{i}<\infty$ and $\alpha_{1}+$ $\cdots \alpha_{m}=\alpha, 1 / p_{1}+\cdots 1 / p_{m}=1 / p, i=1,2, \ldots, m$. If $\omega$ satisfies

$$
\int_{0<t_{1}, \ldots, t_{m}<1}\left(\prod_{i=1}^{m} t_{i}^{\left(\alpha_{i}+n\right) / p_{i}-n}\right) \omega(\vec{t}) d \vec{t}<\infty,
$$

then $\mathscr{G}_{\omega}^{m}$ is bounded from $L^{p_{1}}\left(|x|^{\alpha_{1}} d x\right) \times \cdots \times L^{p_{m}}\left(|x|^{\alpha_{m}} d x\right)$ to $L^{p}\left(|x|^{\alpha} d x\right)$.

Conversely, if $\alpha_{1}=\cdots=\alpha_{m}=(1 / m) \alpha, p_{i}=m p$, $i=1,2, \ldots, m$ and $\mathscr{G}_{\omega}^{m}$ is bounded from $L^{p_{1}}\left(|x|^{\alpha_{1}} d x\right) \times \cdots \times$ $L^{p_{m}}\left(|x|^{\alpha_{m}} d x\right)$ to $L^{p}\left(|x|^{\alpha} d x\right)$, then (37) holds. Moreover, in this case, one has

$$
\begin{aligned}
& \left\|\mathscr{G}_{\omega}^{m}\right\|_{L^{p_{1}}\left(|x|^{\alpha_{1}} d x\right) \times \cdots \times L^{p_{m}}\left(|x|^{\alpha_{m}} d x\right) \rightarrow L^{p}\left(|x|^{\alpha} d x\right)} \\
& \simeq \int_{0<t_{1}, \ldots, t_{m}<1}\left(\prod_{i=1}^{m} t_{i}^{\left(\alpha_{i}+n\right) / p_{i}-n}\right) \omega(\vec{t}) d \vec{t} .
\end{aligned}
$$

\section{Boundedness of $\mathscr{H}_{\omega}^{m}$ on the Product of Morrey-Herz Spaces}

Lets give the second main result of this paper.

Theorem 9. Let $\alpha, \alpha_{1}, \ldots, \alpha_{2} \in \mathbb{R}, \lambda, \lambda_{i}>0,1<p, p_{i}, q, q_{i}<$ $\infty, i=1,2, \ldots, m$, and $\alpha_{1}+\cdots \alpha_{m}=\alpha, 1 / q_{1}+\cdots+1 / q_{m}=1 / q$, $1 / p_{1}+\cdots+1 / p_{m}=1 / p, \lambda_{1}+\cdots+\lambda_{m}=\lambda$. Then $\mathscr{H}_{\omega}^{m}$ is bounded from $M \dot{K}_{p_{1}, q_{1}}^{\alpha_{1}, \lambda_{1}} \times \cdots \times M \dot{K}_{p_{m}, q_{m}}^{\alpha_{m}, \lambda_{m}}$ to $M \dot{K}_{p, q}^{\alpha, \lambda}$ if $\omega$ satisfies

$$
\int_{0<t_{1}, \ldots, t_{m}<1}\left(\prod_{i=1}^{m} t_{i}^{-\left(\alpha_{i}+n / q_{i}-\lambda_{i}\right)}\right) \omega(\vec{t}) d \vec{t}<\infty .
$$

Conversely, when $\alpha_{1}=\cdots=\alpha_{m}=(1 / m) \alpha, \lambda_{1}=\cdots=$ $\lambda_{m}=(1 / m) \lambda, q_{i}=m q$, and $p_{i}=m p, i=1,2, \ldots, m$, if $\mathscr{H}_{\omega}^{m}$ is bounded from $M \dot{K}_{p_{1}, q_{1}}^{\alpha_{1}, \lambda_{1}} \times \cdots \times M \dot{K}_{p_{m}, q_{m}}^{\alpha_{m}, \lambda_{m}}$ to $M \dot{K}_{p, q}^{\alpha, \lambda}$, then (39) holds. And in this case, one has

$$
\begin{aligned}
& \left\|\mathscr{H}_{\omega}^{m}\right\|_{M \dot{K}_{p_{1}, q_{1}}^{\alpha_{1}, \lambda_{1}} \times \cdots \times M \dot{K}_{p_{m}, q_{m}}^{\alpha_{m}, \lambda_{m}} \rightarrow M \dot{K}_{p, q}^{\alpha, \lambda}}^{\alpha,} \\
& \quad \simeq \int_{0<t_{1}, \ldots, t_{m}<1}\left(\prod_{i=1}^{m} t_{i}^{-\left(\alpha_{i}+n / q_{i}-\lambda_{i}\right)}\right) \omega(\vec{t}) d \vec{t} .
\end{aligned}
$$

For the operator $\mathscr{G}_{\omega}^{m}$, we have a corresponding result.

Theorem 10. Let $\alpha, \alpha_{1}, \ldots, \alpha_{2} \in \mathbb{R}, \lambda, \lambda_{i}>0,1<p, p_{i}, q, q_{i}<$ $\infty, i=1,2, \ldots, m$, and $\alpha_{1}+\cdots+\alpha_{m}=\alpha, 1 / q_{1}+\cdots+1 / q_{m}=1 / q$, $1 / p_{1}+\cdots+1 / p_{m}=1 / p, \lambda_{1}+\cdots+\lambda_{m}=\lambda$. Then $\mathscr{G}_{\omega}^{m}$ is bounded from $M \dot{K}_{p_{1}, q_{1}}^{\alpha_{1}, \lambda_{1}} \times \cdots \times M \dot{K}_{p_{m}, q_{m}}^{\alpha_{m}, \lambda_{m}}$ to $M \dot{K}_{p, q}^{\alpha, \lambda}$ if $\omega$ satisfies

$$
\int_{0<t_{1}, \ldots, t_{m}<1}\left(\prod_{i=1}^{m} t_{i}^{\alpha_{i}-\lambda_{i}-n\left(1-1 / q_{i}\right)}\right) \omega(\vec{t}) d \vec{t}<\infty .
$$

Conversely, when $\alpha_{1}=\cdots=\alpha_{m}=(1 / m) \alpha, \lambda_{1}=\cdots=$ $\lambda_{m}=(1 / m) \lambda, q_{i}=m q$, and $p_{i}=m p, i=1,2, \ldots, m$, if $\mathscr{G}_{\omega}^{m}$ is bounded from $M \dot{K}_{p_{1}, q_{1}}^{\alpha_{1}, \lambda_{1}} \times \cdots \times M \dot{K}_{p_{m}, q_{m}}^{\alpha_{m}, \lambda_{m}}$ to $M \dot{K}_{p, q}^{\alpha, \lambda}$, then (41) holds and

$$
\begin{aligned}
& \left.\left\|\mathscr{H}_{\omega}^{m}\right\|_{M \dot{K}_{p_{1}, q_{1}}^{\alpha_{1}, \lambda_{1}} \times \cdots \times M \dot{K}_{p_{m}, q_{m}}^{\alpha_{m}, \lambda_{m}} \rightarrow M \dot{K}_{p, q}^{\alpha, \lambda}}^{\alpha, \lambda}\right) \omega(\vec{t}) d \vec{t} . \\
& \quad \simeq \int_{0<t_{1}, \ldots, t_{m}<1}\left(\prod_{i=1}^{m} t_{i}^{\alpha_{i}-\lambda_{i}-n\left(1-1 / q_{i}\right)}\right.
\end{aligned}
$$

Since the proof of Theorem 10 is similar to the proof of Theorem 9, we give the proof of Theorem 9 only.

Proof of Theorem 9. By similarity, we only consider the case that $m=2$. From the proof of Theorem 5, we know that

$$
\begin{aligned}
\left\|\mathscr{H}_{\omega}^{2}\left(f_{1}, f_{2}\right) \chi_{k}\right\|_{L^{q}\left(\mathbb{R}^{n}\right)} \\
\leq \iint_{0}^{1}\left(\left\|f_{1} \chi_{k+m-1}\right\|_{L^{q_{1}}}+\left\|f_{1} \chi_{k+m}\right\|_{L^{q_{1}}}\right) \\
\quad \times\left(\left\|f_{2} \chi_{k+l-1}\right\|_{L^{q_{2}}}+\left\|f_{2} \chi_{k+l}\right\|_{L^{q_{2}}}\right) \\
\quad \times t_{1}^{-n / q_{1}} t_{2}^{-n / q_{2}} \omega\left(t_{1}, t_{2}\right) d t_{1} d t_{2} .
\end{aligned}
$$

For $1 / p=1 / p_{1}+1 / p_{2}$ and $\alpha=\alpha_{1}+\alpha_{2}, \lambda=\lambda_{1}+\lambda_{2}$, Hölder inequality and Minkowski inequality give

$$
\begin{aligned}
& \left\|\mathscr{H}_{\omega}^{2}\left(f_{1}, f_{2}\right)\right\|_{M \dot{K}_{p, q}^{\alpha, \lambda}} \\
& =\sup _{k_{0} \in \mathbb{Z}} 2^{-k_{0} \lambda}\left(\sum_{k=-\infty}^{k_{0}} 2^{k \alpha p}\left\|\mathscr{H}_{\omega}^{2}\left(f_{1}, f_{2}\right) \chi_{k}\right\|_{L^{q}\left(\mathbb{R}^{n}\right)}^{p}\right)^{1 / p} \\
& \leq \sup _{k_{0} \in \mathbb{Z}} 2^{-k_{0} \lambda}\left(\sum_{k=-\infty}^{k_{0}} 2^{k \alpha p}\right. \\
& \times\left(\iint_{0}^{1}\left(\left\|f_{1} \chi_{k+m-1}\right\|_{L^{q_{1}}}+\left\|f_{1} \chi_{k+m}\right\|_{L^{q_{1}}}\right)\right. \\
& \times\left(\left\|f_{2} \chi_{k+l-1}\right\|_{L^{q_{2}}}+\left\|f_{2} \chi_{k+l}\right\|_{L^{q_{2}}}\right) \\
& \left.\left.\times t_{1}^{-n / q_{1}} t_{2}^{-n / q_{2}} \omega\left(t_{1}, t_{2}\right) d t_{1} d t_{2}\right)^{p}\right)^{1 / p} \\
& \leq \sup _{k_{0} \in \mathbb{Z}} 2^{-k_{0} \lambda} \iint_{0}^{1}\left(\sum _ { k = - \infty } ^ { k _ { 0 } } 2 ^ { k \alpha p } \left(\left\|f_{1} \chi_{k+m-1}\right\|_{L^{q_{1}}}\right.\right. \\
& \left.+\left\|f_{1} \chi_{k+m}\right\|_{L^{q_{1}}}\right)^{p} \\
& \left.\times\left(\left\|f_{2} \chi_{k+l-1}\right\|_{L^{q_{2}}}+\left\|f_{2} \chi_{k+l}\right\|_{L^{q_{2}}}\right)^{p}\right)^{1 / p} \\
& \times t_{1}^{-n / q_{1}} t_{2}^{-n / q_{2}} \omega\left(t_{1}, t_{2}\right) d t_{1} d t_{2}
\end{aligned}
$$


$\leq \sup _{k_{0} \in \mathbb{Z}} 2^{-k_{0} \lambda} \iint_{0}^{1}\left(\sum_{k=-\infty}^{k_{0}} 2^{k \alpha_{1} p_{1}}\left(\left\|f_{1} \chi_{k+m-1}\right\|_{L^{q_{1}}}\right.\right.$ $\left.\left.+\left\|f_{1} \chi_{k+m}\right\|_{L^{q_{1}}}\right)^{p_{1}}\right)^{1 / p_{1}}$

$\times\left(\sum_{k=-\infty}^{k_{0}} 2^{k \alpha_{2} p_{2}}\left(\left\|f_{2} \chi_{k+l-1}\right\|_{L^{q_{2}}}\right.\right.$ $\left.\left.+\left\|f_{2} \chi_{k+l}\right\|_{L^{q_{2}}}\right)^{p_{2}}\right)^{1 / p_{2}}$ $\times t_{1}^{-n / q_{1}} t_{2}^{-n / q_{2}} \omega\left(t_{1}, t_{2}\right) d t_{1} d t_{2}$

$\leq \sup _{k_{0} \in \mathbb{Z}} 2^{-k_{0} \lambda_{1}} \iint_{0}^{1}\left\{\left(\sum_{k=-\infty}^{k_{0}} 2^{k \alpha_{1} p_{1}}\left\|f_{1} \chi_{k+m-1}\right\|_{L^{q_{1}}}^{p_{1}}\right)^{1 / p_{1}}\right.$

$$
\left.+\left(\sum_{k=-\infty}^{k_{0}} 2^{k \alpha_{1} p_{1}}\left\|f_{1} \chi_{k+m}\right\|_{L^{q_{1}}}^{p_{1}}\right)^{1 / p_{1}}\right\}
$$$$
\times \sup _{k_{0} \in \mathbb{Z}} 2^{-k_{0} \lambda_{2}}\left\{\left(\sum_{k=-\infty}^{k_{0}} 2^{k \alpha_{2} p_{2}}\left\|f_{2} \chi_{k+l-1}\right\|_{L^{q_{2}}}^{p_{2}}\right)^{1 / p_{2}}\right.
$$$$
+\left(\sum_{k=-\infty}^{k_{0}} 2^{k \alpha_{2} p_{2}}\right.
$$$$
\left.\left.\times\left\|f_{2} \chi_{k+l}\right\|_{L^{q_{2}}}^{p_{2}}\right)^{1 / p_{2}}\right\}
$$$$
\times t_{1}^{-n / q_{1}} t_{2}^{-n / q_{2}} \omega\left(t_{1}, t_{2}\right) d t_{1} d t_{2}
$$

$\leq\left\|f_{1}\right\|_{M \dot{K}_{p_{1}, q_{1}}^{\alpha_{1}, \lambda_{1}}}\left\|f_{2}\right\|_{M \dot{K}_{p_{2}, q_{2}}^{\alpha_{2}, \lambda_{2}}}$

$\times \iint_{0}^{1}\left(2^{-(m-1) \alpha_{1}}+2^{-m \alpha_{1}}\right)\left(2^{-(l-1) \alpha_{2}}+2^{-l \alpha_{2}}\right)$

$$
\times t_{1}^{-\left(n / q_{1}-\lambda_{1}\right)} t_{2}^{-\left(n / q_{2}-\lambda_{2}\right)} \omega\left(t_{1}, t_{2}\right) d t_{1} d t_{2}
$$

$\leq\left\|f_{1}\right\|_{M \dot{K}_{p_{1}, q_{1}}^{\alpha_{1}, \lambda_{1}}}\left\|f_{2}\right\|_{M \dot{K}_{p_{2}, q_{2}}^{\alpha_{2}, \lambda_{2}}}$

$$
\times \iint_{0}^{1} t_{1}^{-\left(\alpha_{1}+n / q_{1}-\lambda_{1}\right)} t_{2}^{-\left(\alpha_{2}+n / q_{2}-\lambda_{2}\right)} \omega\left(t_{1}, t_{2}\right) d t_{1} d t_{2} .
$$

It means that $\mathscr{H}_{\omega}^{m}$ is bounded from $M \dot{K}_{p_{1}, q_{1}}^{\alpha_{1}, \lambda_{1}} \times \cdots \times M \dot{K}_{p_{m}, q_{m}}^{\alpha_{m}, \lambda_{m}}$ to $M \dot{K}_{p, q}^{\alpha, \lambda}$.
On the other hand, we define

$$
\begin{array}{ll}
f_{1}(x)=|x|^{-\left(\alpha_{1}+n / q_{1}-\lambda_{1}\right)}, & x \in \mathbb{R}^{n}, \\
f_{2}(x)=|x|^{-\left(\alpha_{2}+n / q_{2}-\lambda_{2}\right)}, & x \in \mathbb{R}^{n} .
\end{array}
$$

When $\alpha_{1} \neq \lambda_{1}$ and $\alpha_{2} \neq \lambda_{2}$, we get

$$
\begin{aligned}
\left\|f_{1} \chi_{k}\right\|_{L^{q_{1}}} & =\left(\int_{2^{k-1} \leq|x|<2^{k}}|x|^{-\left(\alpha_{1}+n / q_{1}-\lambda_{1}\right) q_{1}} d x\right)^{1 / q_{1}} \\
& =2^{-\left(\alpha_{1}-\lambda_{1}\right) k}\left|\frac{S_{n}\left(2^{\left(\alpha_{1}-\lambda_{1}\right) q_{1}}-1\right)}{\left(\alpha_{1}-\lambda_{1}\right) q_{1}}\right|^{1 / q_{1}},
\end{aligned}
$$

where $S_{n}=n \pi^{n / 2} / \Gamma(1+n / 2)$. Thus

$\left\|f_{1}\right\|_{M \dot{K}_{p_{1}, q_{1}}^{\alpha_{1}, \lambda_{1}}}$

$$
=2^{\lambda_{1}}\left(\frac{1}{2^{\lambda_{1} p_{1}}-1}\right)^{1 / p_{1}}\left|\frac{S_{n}\left(2^{\left(\alpha_{1}-\lambda_{1}\right) q_{1}}-1\right)}{\left(\alpha_{1}-\lambda_{1}\right) q_{1}}\right|^{1 / q_{1}} .
$$

It is similar to obtain

$\left\|f_{2}\right\|_{M \dot{K}_{p_{2}, q_{2}}^{\alpha_{2}, \lambda_{2}}}$

$$
=2^{\lambda_{2}}\left(\frac{1}{2^{\lambda_{2} p_{2}}-1}\right)^{1 / p_{2}}\left|\frac{S_{n}\left(2^{\left(\alpha_{2}-\lambda_{2}\right) q_{2}}-1\right)}{\left(\alpha_{2}-\lambda_{2}\right) q_{2}}\right|^{1 / q_{2}} .
$$

For $\alpha=\alpha_{1}+\alpha_{2}, \lambda=\lambda_{1}+\lambda_{2}$, and $1 / q=1 / q_{1}+1 / q_{2}$, we have

$$
\begin{aligned}
& \mathscr{H}_{\omega}^{2}\left(f_{1}, f_{2}\right)(x) \\
& =|x|^{-(\alpha+n / q-\lambda)} \iint_{0}^{1} t_{1}^{-\left(\alpha_{1}+n / q_{1}-\lambda_{1}\right)} t_{2}^{-\left(\alpha_{2}+n / q_{2}-\lambda_{2}\right)} \\
& \quad \times \omega\left(t_{1}, t_{2}\right) d t_{1} d t_{2} .
\end{aligned}
$$

By simple computation, we get

$$
\begin{aligned}
& \left\|\mathscr{H}_{\omega}^{2}\left(f_{1}, f_{2}\right) \chi_{k}\right\|_{L^{q}\left(\mathbb{R}^{n}\right)}^{p} \\
& =\left(\int_{\mathbb{R}^{n}}\left|\mathscr{H}_{\omega}^{2}\left(f_{1}, f_{2}\right)(x)\right|^{q} \chi_{k}(x) d x\right)^{p / q} \\
& =\left(\int_{\mathbb{R}^{n}}|x|^{-(\alpha+n / q-\lambda) q} \chi_{k}(x)\right. \\
& \times\left(\iint_{0}^{1} t_{1}^{-\left(\alpha_{1}+n / q_{1}-\lambda_{1}\right)} t_{2}^{-\left(\alpha_{2}+n / q_{2}-\lambda_{2}\right)}\right. \\
& =\left(\int_{2^{k-1} \leq|x|<2^{k}}|x|^{-\left(\alpha+n / q^{-\lambda) q}\right.} d x\right)^{p / q} \\
& \quad \times\left(\iint_{0}^{1} t_{1}^{-\left(\alpha_{1}+n / q_{1}-\lambda_{1}\right)} t_{2}^{-\left(\alpha_{2}+n / q_{2}-\lambda_{2}\right)}\right)^{p / q} \\
&
\end{aligned}
$$

$$
\left.\times \omega\left(t_{1}, t_{2}\right) d t_{1} d t_{2}\right)^{p}
$$




$$
\begin{gathered}
=2^{-(\alpha-\lambda) k p}\left|\frac{S_{n}\left(2^{(\alpha-\lambda) q}-1\right)}{(\alpha-\lambda) q}\right|^{p / q} \\
\times\left(\iint_{0}^{1} t_{1}^{-\left(\alpha_{1}+n / q_{1}-\lambda_{1}\right)} t_{2}^{-\left(\alpha_{2}+n / q_{2}-\lambda_{2}\right)}\right. \\
\left.\times \omega\left(t_{1}, t_{2}\right) d t_{1} d t_{2}\right)^{p} .
\end{gathered}
$$

Since $\alpha_{1}=\alpha_{2}=(1 / 2) \alpha, p_{1}=p_{2}=2 p, q_{1}=q_{2}=2 q$, and $\lambda_{1}=\lambda_{2}=(1 / 2) \lambda$, we have

$$
\begin{aligned}
& \left\|\mathscr{H}_{\omega}^{2}\left(f_{1}, f_{2}\right)\right\|_{M \dot{K}_{p, q}^{\alpha, \lambda}} \\
& =\sup _{k_{0} \in \mathbb{Z}} 2^{-k_{0} \lambda}\left(\sum_{k=-\infty}^{k_{0}} 2^{k \alpha p}\left\|\mathscr{H}_{\omega}^{2}\left(f_{1}, f_{2}\right) \chi_{k}\right\|_{L^{q}\left(\mathbb{R}^{n}\right)}^{p}\right)^{1 / p} \\
& =\sup _{k_{0} \in \mathbb{Z}} 2^{-k_{0} \lambda}\left(\sum_{k=-\infty}^{k_{0}} 2^{k \alpha p} 2^{-(\alpha-\lambda) k p}\right)^{1 / p} \\
& \times\left|\frac{S_{n}\left(2^{(\alpha-\lambda) q}-1\right)}{(\alpha-\lambda) q}\right|^{1 / q} \\
& \times \iint_{0}^{1} t_{1}^{-\left(\alpha_{1}+n / q_{1}-\lambda_{1}\right)} t_{2}^{-\left(\alpha_{2}+n / q_{2}-\lambda_{2}\right)} \\
& \times \omega\left(t_{1}, t_{2}\right) d t_{1} d t_{2} \\
& =2^{\lambda}\left(\frac{1}{2^{\lambda p}-1}\right)^{1 / p}\left|\frac{S_{n}\left(2^{(\alpha-\lambda) q}-1\right)}{(\alpha-\lambda) q}\right|^{1 / q} \\
& \times \iint_{0}^{1} t_{1}^{-\left(\alpha_{1}+n / q_{1}-\lambda_{1}\right)} t_{2}^{-\left(\alpha_{2}+n / q_{2}-\lambda_{2}\right)} \\
& \times \omega\left(t_{1}, t_{2}\right) d t_{1} d t_{2} \\
& =\left\|f_{1}\right\|_{M \dot{K}_{p_{1}, q_{1}}^{\alpha_{1}, \lambda_{1}}}\left\|f_{2}\right\|_{M \dot{K}_{p_{2}, q_{2}}^{\alpha_{2}, \lambda_{2}}} \\
& \times \iint_{0}^{1} t_{1}^{-\left(\alpha_{1}+n / q_{1}-\lambda_{1}\right)} t_{2}^{-\left(\alpha_{2}+n / q_{2}-\lambda_{2}\right)} \\
& \times \omega\left(t_{1}, t_{2}\right) d t_{1} d t_{2} .
\end{aligned}
$$

Thus, (39) holds in this case.

When $\alpha_{1}=\lambda_{1}$ and $\alpha_{2}=\lambda_{2}$, it is easy to obtain

$$
\begin{aligned}
\left\|f_{1} \chi_{k}\right\|_{L^{q_{1}}}^{q_{1}} & =\left\|f_{2} \chi_{k}\right\|_{L^{q_{2}}}^{q_{2}}=\int_{2^{k-1} \leq|x|<2^{k}}|x|^{-n} d x \\
& =S_{n} \ln 2 .
\end{aligned}
$$

Thus, we have

$$
\begin{aligned}
& \left\|f_{1}\right\|_{M \dot{K}_{p_{1}, q_{1}}^{\alpha_{1}, \lambda_{1}}}=2^{\lambda_{1}}\left(\frac{1}{2^{\lambda_{1} p_{1}}-1}\right)^{1 / p_{1}}\left(S_{n} \ln 2\right)^{1 / q_{1}}, \\
& \left\|f_{2}\right\|_{M \dot{K}_{p_{2}, q_{2}}^{\alpha_{2}, \lambda_{2}}}=2^{\lambda_{2}}\left(\frac{1}{2^{\lambda_{2} p_{2}}-1}\right)^{1 / p_{2}}\left(S_{n} \ln 2\right)^{1 / q_{2}} .
\end{aligned}
$$

So

$$
\begin{aligned}
& \mathscr{H}_{\omega}^{2}\left(f_{1}, f_{2}\right)(x) \\
& \quad=|x|^{-n / q} \iint_{0}^{1} t_{1}^{-n / q_{1}} t_{2}^{-n / q_{2}} \omega\left(t_{1}, t_{2}\right) d t_{1} d t_{2} .
\end{aligned}
$$

Then, we get

$$
\begin{aligned}
& \left\|\mathscr{H}_{\omega}^{2}\left(f_{1}, f_{2}\right) \chi_{k}\right\|_{L^{q}\left(\mathbb{R}^{n}\right)} \\
& \quad=\left(S_{n} \ln 2\right)^{1 / q} \iint_{0}^{1} t_{1}^{-n / q_{1}} t_{2}^{-n / q_{2}} \omega\left(t_{1}, t_{2}\right) d t_{1} d t_{2} .
\end{aligned}
$$

Thus,

$$
\begin{aligned}
& \left\|\mathscr{H}_{\omega}^{2}\left(f_{1}, f_{2}\right)\right\|_{M \dot{K}_{p, q}^{\alpha, \lambda}} \\
& =\sup _{k_{0} \in \mathbb{Z}} 2^{-k_{0} \lambda}\left(\sum_{k=-\infty}^{k_{0}} 2^{k \alpha p}\left\|\mathscr{H}_{\omega}^{2}\left(f_{1}, f_{2}\right) \chi_{k}\right\|_{L^{q}\left(\mathbb{R}^{n}\right)}^{p}\right)^{1 / p} \\
& =\sup _{k_{0} \in \mathbb{Z}} 2^{-k_{0} \lambda}\left(\sum_{k=-\infty}^{k_{0}} 2^{k \alpha p}\left(S_{n} \ln 2\right)^{p / q}\right. \\
& \times\left(\iint_{0}^{1} t_{1}^{-n / q_{1}} t_{2}^{-n / q_{2}}\right. \\
& \left.\left.\left.=\sup _{k_{0} \in \mathbb{Z}} 2^{-k_{0} \lambda}\left(\sum_{k=-\infty}^{k_{0}} 2^{k \alpha p}\right)^{1 / p}\left(t_{1}, t_{2}\right) d t_{1} d t_{2}\right)^{1 / p}\right)^{1 / p}\right)^{1 / q} \\
& \quad \times \iint_{0}^{1} t_{1}^{-n / q_{1}} t_{2}^{-n / q_{2}} \omega\left(t_{1}, t_{2}\right) d t_{1} d t_{2} .
\end{aligned}
$$

Since $\alpha_{1}=\lambda_{1}$ and $\alpha_{2}=\lambda_{2}$, we get $\alpha=\lambda$. Thus

$$
\begin{aligned}
\| \mathscr{H}_{\omega}^{2}( & \left.f_{1}, f_{2}\right) \|_{M \dot{K}_{p, q}^{\alpha, \lambda}} \\
= & 2^{\lambda}\left(\frac{1}{2^{\lambda p}-1}\right)^{1 / p}\left(S_{n} \ln 2\right)^{1 / q} \\
& \times \iint_{0}^{1} t_{1}^{-n / q_{1}} t_{2}^{-n / q_{2}} \omega\left(t_{1}, t_{2}\right) d t_{1} d t_{2} \\
= & \left\|f_{1}\right\|_{M \dot{K}_{p_{1}, q_{1}}^{\alpha_{1}, \lambda_{1}}}\left\|f_{2}\right\|_{M \dot{K}_{p_{2}, q_{2}}^{\alpha_{2}, \lambda_{2}}} \\
& \times \iint_{0}^{1} t_{1}^{-n / q_{1}} t_{2}^{-n / q_{2}} \omega\left(t_{1}, t_{2}\right) d t_{1} d t_{2} .
\end{aligned}
$$

This tells us that (39) also holds in this case. 
Furthermore, when one of $\alpha_{1}=\lambda_{1}, \alpha_{2}=\lambda_{2}$ holds, we suppose $\alpha_{1}=\lambda_{1}$ but $\alpha_{2} \neq \lambda_{2}$ here. From the above computation, we have

$$
\begin{gathered}
\left\|f_{1}\right\|_{M \dot{K}_{p_{1}, q_{1}}^{\alpha_{1}, \lambda_{1}}}=2^{\lambda_{1}}\left(\frac{1}{2^{\lambda_{1} p_{1}}-1}\right)^{1 / p_{1}}\left(S_{n} \ln 2\right)^{1 / q_{1}}, \\
\left\|f_{2}\right\|_{M \dot{K}_{p_{2}, q_{2}}^{\alpha_{2}, \lambda_{2}}} \\
\quad=2^{\lambda_{2}}\left(\frac{1}{2^{\lambda_{2} p_{2}}-1}\right)^{1 / p_{2}}\left|\frac{S_{n}\left(2^{\left(\alpha_{2}-\lambda_{2}\right) q_{2}}-1\right)}{\left(\alpha_{2}-\lambda_{2}\right) q_{2}}\right|^{1 / q_{2}} .
\end{gathered}
$$

It gives

$$
\begin{aligned}
& \mathscr{H}_{\omega}^{2}\left(f_{1}, f_{2}\right)(x) \\
& =|x|^{-\left(\alpha_{2}+(n / q)-\lambda_{2}\right)} \iint_{0}^{1} t_{1}^{-n / q_{1}} t_{2}^{-\left(\alpha_{2}+n / q_{2}-\lambda_{2}\right)} \\
& \quad \times \omega\left(t_{1}, t_{2}\right) d t_{1} d t_{2} .
\end{aligned}
$$

So, we have

$$
\begin{aligned}
&\left\|\mathscr{H}_{\omega}^{2}\left(f_{1}, f_{2}\right)\right\|_{M \dot{K}_{p, q}^{\alpha, \lambda}} \sup _{k_{0} \in \mathbb{Z}} 2^{-k_{0} \lambda}\left(\sum_{k=-\infty}^{k_{0}} 2^{k \alpha p}\left\|\mathscr{H}_{\omega}^{2}\left(f_{1}, f_{2}\right) \chi_{k}\right\|_{L^{q}\left(\mathbb{R}^{n}\right)}^{p}\right)^{1 / p} \\
&=\sup _{k_{0} \in \mathbb{Z}} 2^{-k_{0} \lambda}\left(\sum_{k=-\infty}^{k_{0}} 2^{k \alpha p} 2^{-\left(\alpha_{2}-\lambda_{2}\right) k p}\right)^{1 / p} \\
& \quad \times\left|\frac{S_{n}\left(2^{\left(\alpha_{2}-\lambda_{2}\right) q}-1\right)}{\left(\alpha_{2}-\lambda_{2}\right) q}\right|^{1 / q} \\
& \quad \times \iint_{0}^{1} t_{1}^{-n / q_{1}} t_{2}^{-\left(\alpha_{2}+n / q_{2}-\lambda_{2}\right)} \omega\left(t_{1}, t_{2}\right) d t_{1} d t_{2} \\
&=2^{\lambda}\left(\frac{1}{2^{\lambda p}-1}\right)^{1 / p}\left|\frac{S_{n}\left(2^{\left(\alpha_{2}-\lambda_{2}\right) q}-1\right)}{\left(\alpha_{2}-\lambda_{2}\right) q}\right|^{1 / q} \\
& \quad \times \iint_{0}^{1} t_{1}^{-n / q_{1}} t_{2}^{-\left(\alpha_{2}+n / q_{2}-\lambda_{2}\right)} \omega\left(t_{1}, t_{2}\right) d t_{1} d t_{2} .
\end{aligned}
$$

Since $\alpha_{1}=\alpha_{2}=(1 / 2) \alpha, p_{1}=p_{2}=2 p, q_{1}=q_{2}=2 q$, and $\lambda_{1}=\lambda_{2}=(1 / 2) \lambda$, using Taylor expansion yields

$$
\begin{aligned}
& \left|\frac{S_{n}\left(2^{\left(\alpha_{2}-\lambda_{2}\right) q}-1\right)}{\left(\alpha_{2}-\lambda_{2}\right) q}\right|^{1 / q} \\
& \quad \geq\left(S_{n} \ln 2\right)^{1 / q_{1}}\left|\frac{S_{n}\left(2^{\left(\alpha_{2}-\lambda_{2}\right) q_{2}}-1\right)}{\left(\alpha_{2}-\lambda_{2}\right) q_{2}}\right|^{1 / q_{2}} .
\end{aligned}
$$

Thus, we have

$$
\begin{aligned}
& \left\|\mathscr{H}_{\omega}^{2}\left(f_{1}, f_{2}\right)\right\|_{M \dot{K}_{p, q}^{\alpha, \lambda}} \\
& \geq\left\|f_{1}\right\|_{M \dot{K}_{p_{1}, q_{1}}^{\alpha_{1}, \lambda_{1}}}\left\|f_{2}\right\|_{M \dot{K}_{p_{2}, q_{2}}^{\alpha_{2}, \lambda_{2}}} \\
& \quad \times \iint_{0}^{1} t_{1}^{-n / q_{1}} t_{2}^{-\left(\alpha_{2}+n / q_{2}-\lambda_{2}\right)} \omega\left(t_{1}, t_{2}\right) d t_{1} d t_{2} .
\end{aligned}
$$

The above inequality gives

$$
\begin{aligned}
& \left\|\mathscr{H}_{\omega}^{m}\right\|_{M \dot{K}_{p_{1}, q_{1}}^{\alpha_{1}, \lambda_{1}}{ }^{\prime} \cdots \times M \dot{K}_{P m}, q_{m}}^{\alpha_{m}, \lambda_{m}} \rightarrow M \dot{K}_{p, q}^{\alpha, \lambda} \\
& \quad \geq \int_{0<t_{1}, \ldots, t_{m}<1}\left(\prod_{i=1}^{m} t_{i}^{-\left(\alpha_{i}+n / q_{i}-\lambda_{i}\right)}\right) \omega(\vec{t}) d \vec{t} .
\end{aligned}
$$

Since $\mathscr{H}_{\omega}^{m}$ is bounded from $M \dot{K}_{p_{1}, q_{1}}^{\alpha_{1}, \lambda_{1}} \times \cdots \times M \dot{K}_{p_{m}, q_{m}}^{\alpha_{m}, \lambda_{m}}$ to $M \dot{K}_{p, q}^{\alpha, \lambda}$, we know that (39) holds and

$$
\begin{aligned}
& \left\|\mathscr{H}_{\omega}^{m}\right\|_{M \dot{K}_{p_{1}, q_{1}}^{\alpha_{1}, \lambda_{1}} \times \cdots \times M \dot{K}_{P m}, q_{m}}^{\alpha_{m}, \lambda_{m}} \rightarrow M \dot{K}_{p, q}^{\alpha, \lambda} \\
& \quad \simeq \int_{0<t_{1}, \ldots, t_{m}<1}\left(\prod_{i=1}^{m} t_{i}^{-\left(\alpha_{i}+n / q_{i}-\lambda_{i}\right)}\right) \omega(\vec{t}) d \vec{t} .
\end{aligned}
$$

The proof of Theorem 9 is complete.

\section{Conflict of Interests}

The authors declare that there is no conflict of interests regarding the publication of this paper.

\section{Acknowledgments}

This work was partially supported by NSF of China (Grant nos. 11271175, 11271162, and 61304023), the AMEP, and DYSP of Linyi University.

\section{References}

[1] G. H. Hardy, J. E. Littlewood, and G. Polya, Inequalities, Cambridge University Press, London, UK, 2nd edition, 1952.

[2] G. H. Hardy, J. E. Littlewood, and G. Polya, Inequalities, Cambridge University Press, London, UK, 1934.

[3] A. Kufner and L. E. Persson, Weighted Inequalities of Hardy Type, World Scientific, Singapore, 2003.

[4] C. Carton-Lebrun and M. Fosset, "Moyennes et quotients de Taylor dans BMO," Bulletin de la Société Royale des Sciences de Liege, vol. 53, pp. 85-87, 1984.

[5] J. Xiao, " $L^{p}$ and BMO bounds of weighted Hardy-Littlewood averages," Journal of Mathematical Analysis and Applications, vol. 262, no. 2, pp. 660-666, 2001.

[6] Z. W. Fu, S. Z. Lu, and W. Yuan, "A weighted variant of Riemann-Liouville fractional integral on $R_{n}$," Abstract and Applied Analysis, vol. 2012, Article ID 780132, 18 pages, 2012.

[7] Z. Fu and S. Lu, "Weighted Hardy operators and commutators on Morrey spaces," Frontiers of Mathematics in China, vol. 5, no. 3, pp. 531-539, 2010.

[8] S. Z. Lu, D. C. Yang, and G. E. Hu, Herz Type Spaces and Their Applications, Science Press, Beijing, China, 2008. 
[9] J. Wu, "Boundedness of multilinear singular integral operators on the homogeneous Morrey-Herz spaces," Ukrainian Mathematical Journal, vol. 61, no. 10, pp. 1684-1693, 2009.

[10] C. Morrey, "On the solutions of quasi-linear elliptic partial differential equations," Transactions of the American Mathematical Society, vol. 43, pp. 126-166, 1938.

[11] W. Yuan, W. Sickel, and D. Yang, "Morrey and Campanato meet Besov, Lizorkin and Triebel," Lecture Notes in Mathematics, vol. 2005, pp. 1-292, 2010.

[12] Z. G. Liu and Z. W. Fu, "Weighted Hardy-Littlewood averages on Herz spaces," Acta Mathematics Sinica, vol. 49, pp. 10851090, 2006.

[13] Z. W. Fu and S. Z. Lu, "A remark on weighted Hardy-Littlewood averages on Herz spaces," Advances in Mathematics, vol. 37, pp. 632-636, 2008 (Chinese).

[14] R. R. Coifman and Y. Meyer, "On commutators of singular integrals and bilinear singular integrals," Transactions of the American Mathematical Society, vol. 212, pp. 315-331, 1975.

[15] D. Fan and X. Li, "A bilinear oscillatory integral along parabolas," Positivity, vol. 13, no. 2, pp. 339-366, 2009.

[16] L. Grafakos and X. Li, "Uniform bounds for the bilinear Hilbert transforms, I," Annals of Mathematics, vol. 159, no. 3, pp. 889933, 2004.

[17] X. Li, "Uniform bounds for the bilinear hilbert transforms, II," Revista Matematica Iberoamericana, vol. 22, no. 3, pp. 10691126, 2006.

[18] Z. W. Fu, L. Grafakos, S. Z. Lu, and F. Y. Zhao, "Sharp bounds for m-linear Hardy and Hilbert operators," Houston Journal of Mathematics, vol. 38, pp. 225-244, 2012.

[19] Z. W. Fu, S. L. Gong, S. Z. Lu, and W. Yuan, "Weighted multilinear Hardy operators and commutators," Forum Mathematicum. In press. 


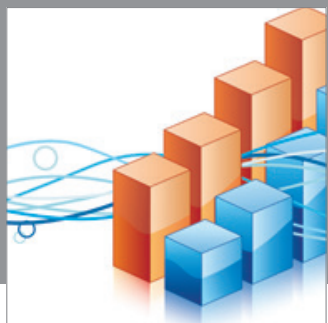

Advances in

Operations Research

mansans

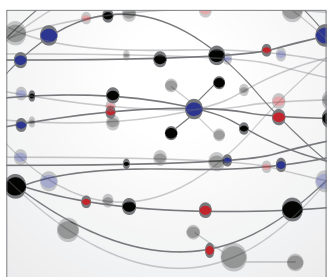

The Scientific World Journal
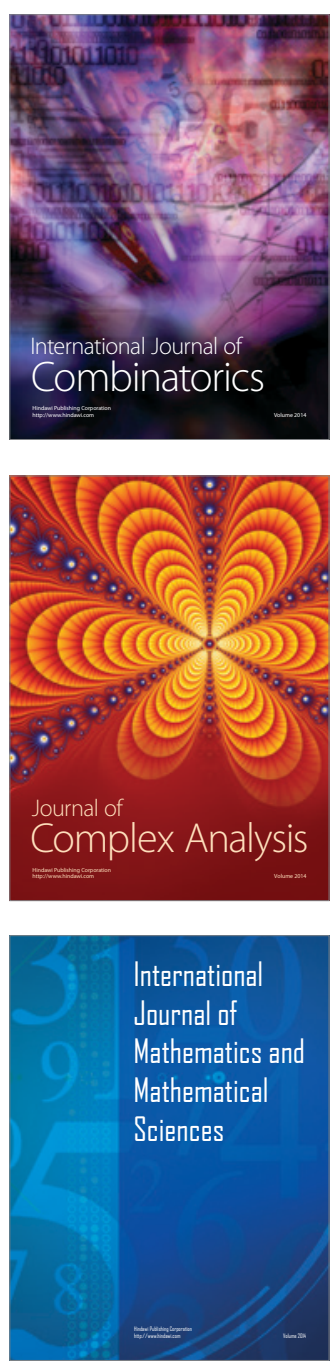
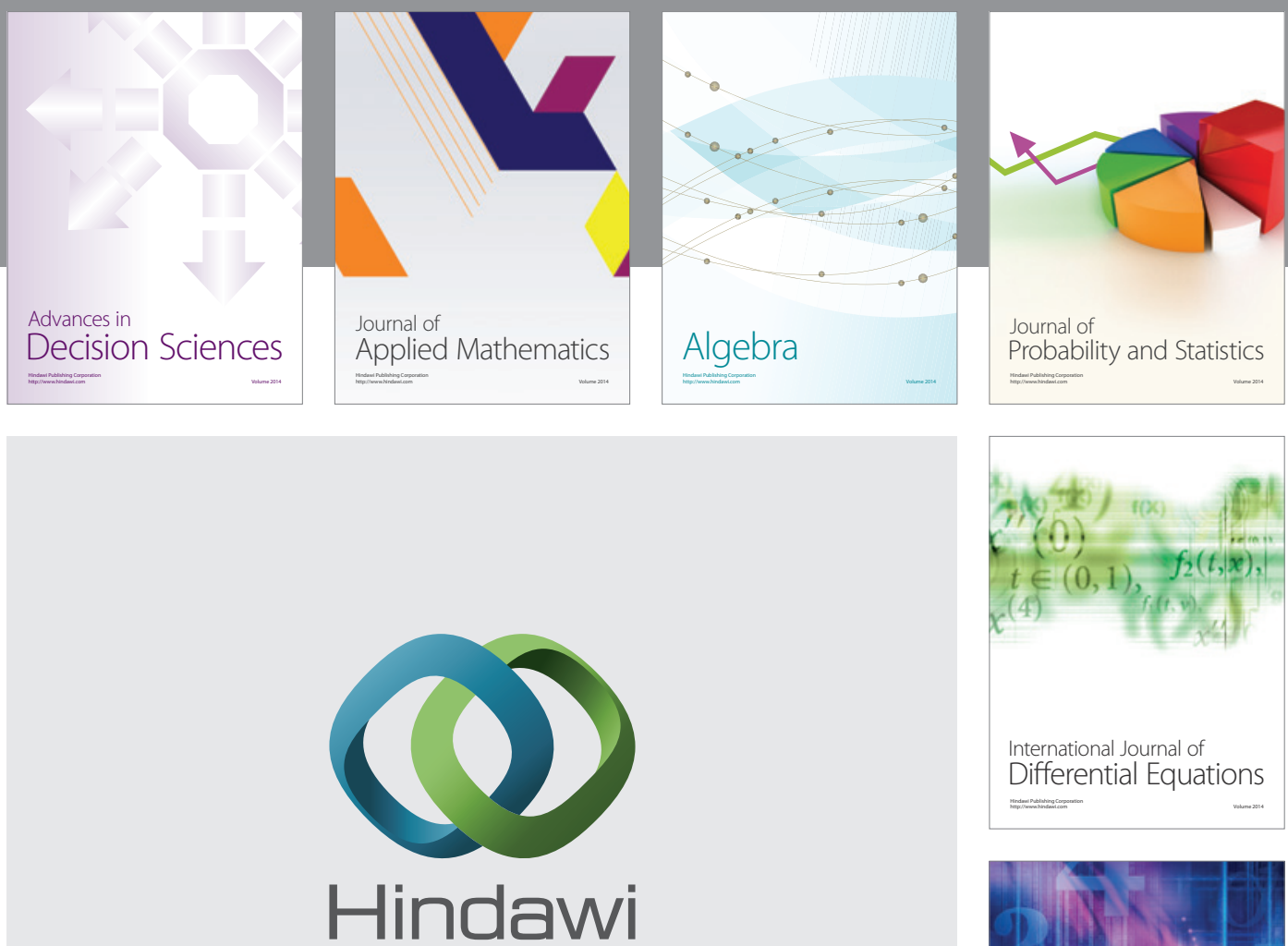

Submit your manuscripts at http://www.hindawi.com
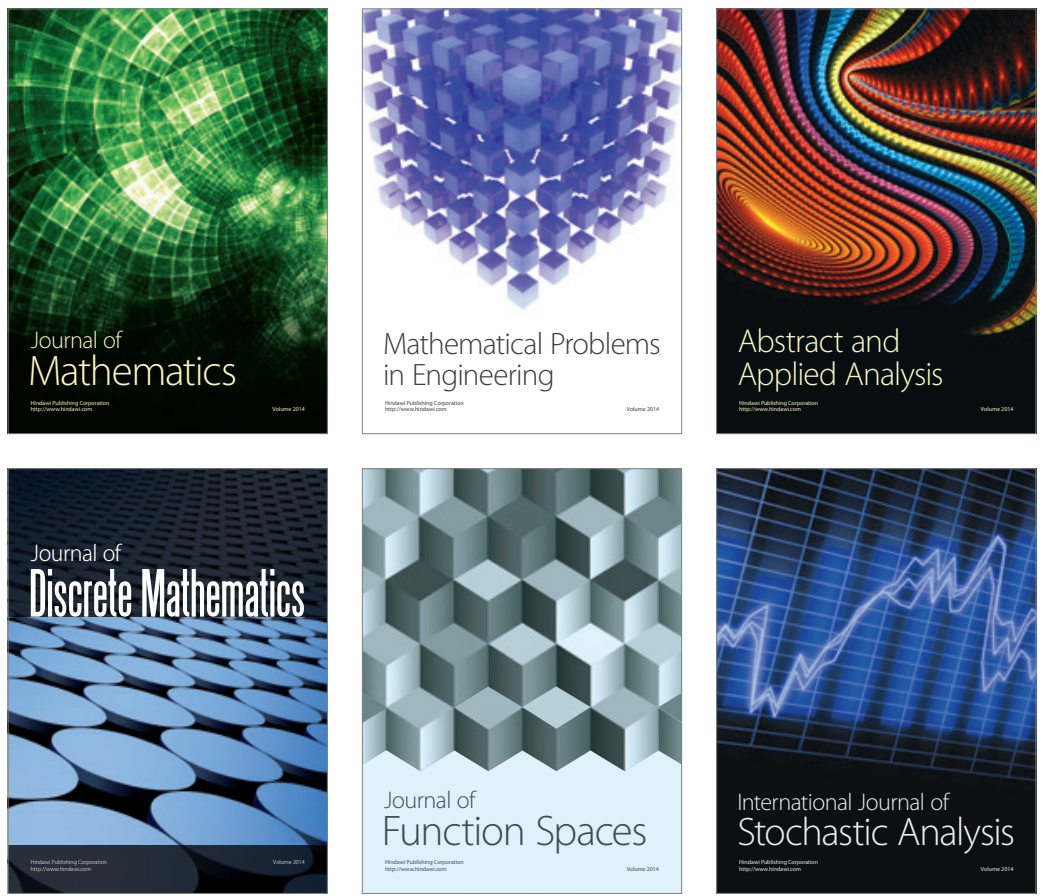

Journal of

Function Spaces

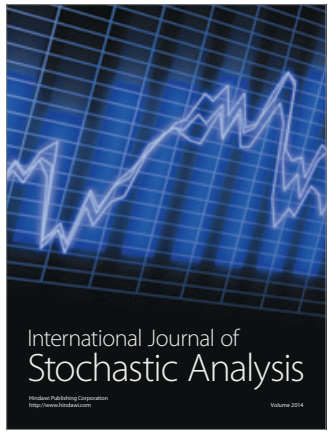

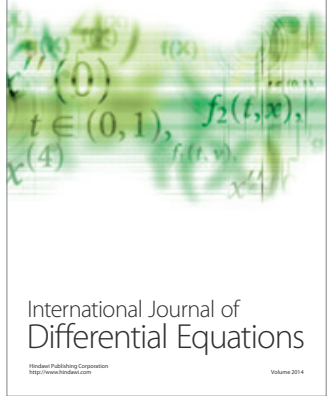
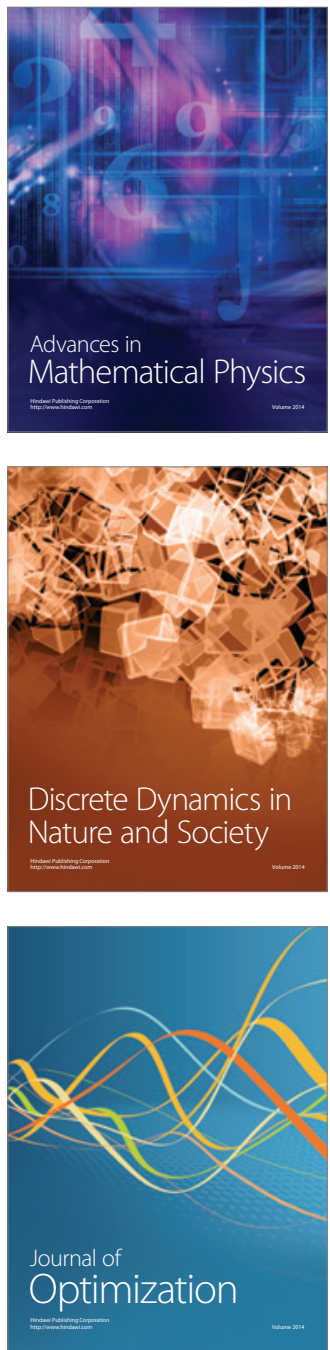